\title{
Validation of the Load-Resilient Ion Cyclotron Resonance Frequency Antenna Concept on Tore Supra Plasmas
}

\author{
K. Vulliez, A. Argouarch, G. Bosia, G. Berger-By, S. Bremond, L. Colas, G. Lombard, A. \\ Mendes L. Millon, P. Mollard, D. Volpe, B. Beaumont, A. Bécoulet, F. Clairet, A. Ekedahl, \\ M. Elkhaldi, J. Gunn, G.T. Hoang and the Tore Supra Team. \\ Association EURATOM-CEA, CEA/DSM/Institut de Recherche sur la Fusion par confinement Magnétique, \\ CEA Cadarache, F-13108 St. Paul lez Durance, France
}

Abstract: $n$ the framework of the ion cyclotron resonance frequency (ICRF) heating development at CEA Cadarache, a prototype antenna based on the load-resilient electrical layout foreseen for ITER has been built. This prototype was recently tested in Tore Supra. The ITER-like electrical scheme has been validated during fast perturbations at the edge plasma. Clear load resilience properties are reported. Main conclusions and consequences to be learned for the development of ITER antenna are discussed.

\section{INTRODUCTION}

ICRF wave coupling is one of critical issues in the H-mode environment, thus in the ITER baseline scenario. In H-mode plasmas, the Edge Localized Modes (ELMs) create sudden increases of antenna coupling which, if not compensated by the electrical circuit, creates a mismatch repetitively tripping the generators. Several techniques have been implemented in ASDEX Upgrade [1] and JET [2] to avoid this problem.

The development of an ITER-Like prototype (ILP) ICRF antenna for Tore Supra (TS) was initiated in 2003 [3]. A first prototype was previously tested in 2004 at moderate power level and pulse length $(500 \mathrm{~kW} / 6 \mathrm{~s})$ [4]. These experiments provided the first indications on the load tolerant properties of the electrical circuit and data on the antenna operation and global radio-frequency aspects. However, the operation of this prototype was quickly terminated by destructive damage to the internal matching components. This first prototype was then modified and significantly improved; including the implementation of diagnostic and control systems for the automatic control loops and the matching experiments. These modifications were based on the fruitful lessons learned during the 2004 campaign and the observations made during disassembly of the antenna [5]. This paper reports on the encouraging results obtained during this new campaign of tests, with emphasis on load tolerance properties under fast perturbations of the plasma facing the antenna. The paper is organized as follows. The global description of the prototype antenna and latest improvements are reported in Section II. Section III reports the experiments using the modified prototype. The know-how and experience gained from these experiments are discussed in Section IV.

\section{GLOBAL DESCRIPTION OF THE ANTENNA AND LATEST ENHANCEMENTS}

The prototype launcher (Fig. 1) consists of two ITER-like Resonant Double Loop (RDL) antennae arranged toroidally. Each antenna is composed of two poloidal current straps short-circuited on one side on the Faraday Screen (FS) and connected in series to a variable capacitor at the other one. The tuning unit [6] composed of two capacitors, connected side by side, is fed by a two stage quarter-wave length impedance transformers (conversion from the $30 \Omega$ feeding line to the $4 \Omega$ of antenna/tuning assembly). Compared to the "conventional" RDL circuit, this RF circuit has the advantage that the antenna sensitivity at high variation of the resistive part of the load is lower, feature enhanced by the straps short in length $(\sim / 0.3 \mathrm{~m})$. 


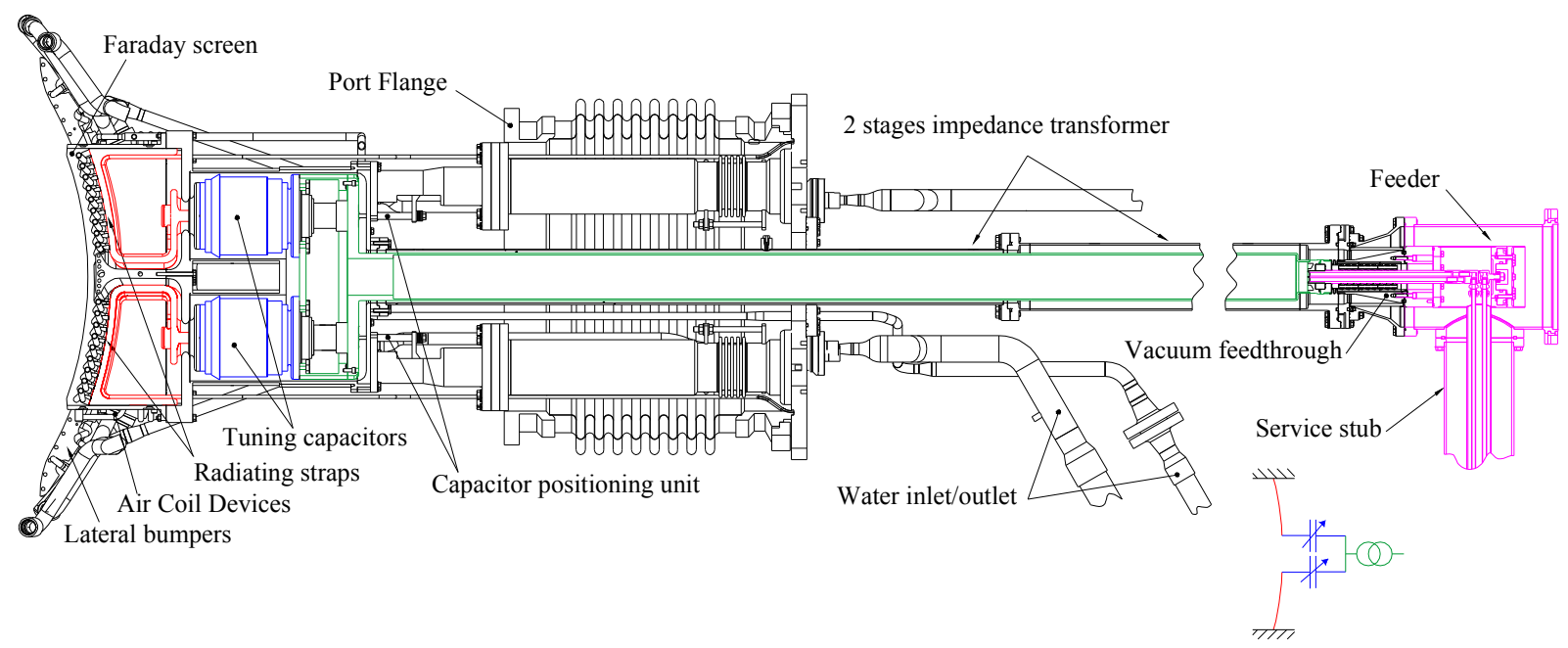

Figure 1: Layout of the ITER-like antenna prototype tested in 2004, featuring in color the different subsystems; red: radiating straps; green : vacuum feed $(9 \Omega)$ and 2-stage impedance transformer $(9 / 20 \Omega)$; blue: tuning capacitors ; magenta : quarter wavelength stub.

From the 2004 campaign several enhancements of the prototype were made. The first was the addition of corona rings around the brazed joints of capacitor, to prevent arcing in this sensitive region. Based on arcs traces observed during the dismounting of the antenna, some "sharp" edges, found in localized areas on the low impedance parts, were smoothed off to improve the voltage standoff capability. A massive poloidal septum was also built (Fig. 2b) to replace the original recessed one (Fig. 2a) to reduce the toroidal mutual coupling between adjacent straps.

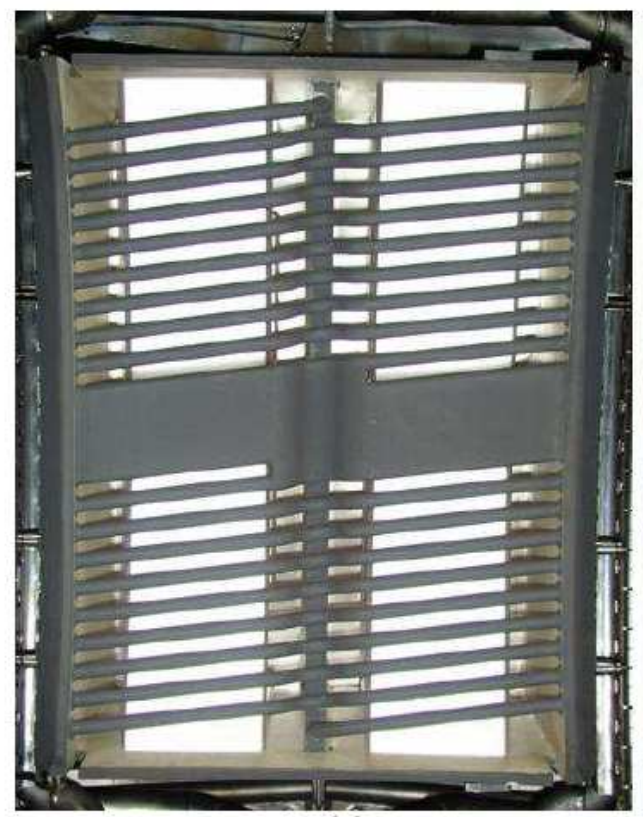

(a)

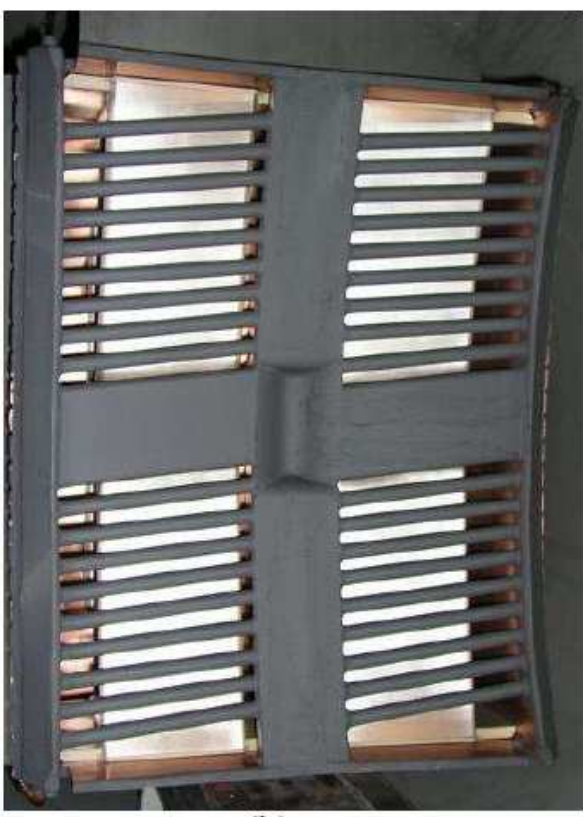

(b)

Figure 2: (a) Previous prototype: Faraday screen with a recessed septum; (b) Present prototype: New Faraday screen with the new massive poloidal septum.

RF current measurements in the resonant part of the RF circuit have also been implemented for strap current control. Associated with a dedicated feedback control scheme [7], they allow the $2 \times 2$ array match point to be found automatically in dipole or monopole toroidal phasing. The new RF measurements are also more sensitive to the presence of arcs than previous ones, which were located in the feeding lines and proved inefficient in detecting 
breakdowns in the prototype [8]. In addition to enhancements of the RF circuit, diagnostics and protection systems were added or improved. An arc safety system based on SubHarmonic Arc Detection (SHAD), in a frequency band lower than the generator operation frequency, was implemented on the launcher [9]. This system works in parallel with a classical safety system based on Vreflected/Vforward signals.

\section{EXPERIMENTS ON TORE SUPRA PLASMAS}

A start-up phase of few days was necessary to commission and find the antenna match points on the machine vacuum, prior to the plasma experiments. The experimental phase on plasma then began, following four major lines: (1) set-up of matching points on plasma and qualification of the numerical model and matching algorithm; (2) study of the load resilience properties of the system; (3) comparison of the ILP with the classical RDL antenna [10], regarding the sheath potentials and Faraday Screen hot spots, interaction with lower hybrid launchers, quick variation of plasma conditions; (4) investigation of the power limits of the launcher.

a)

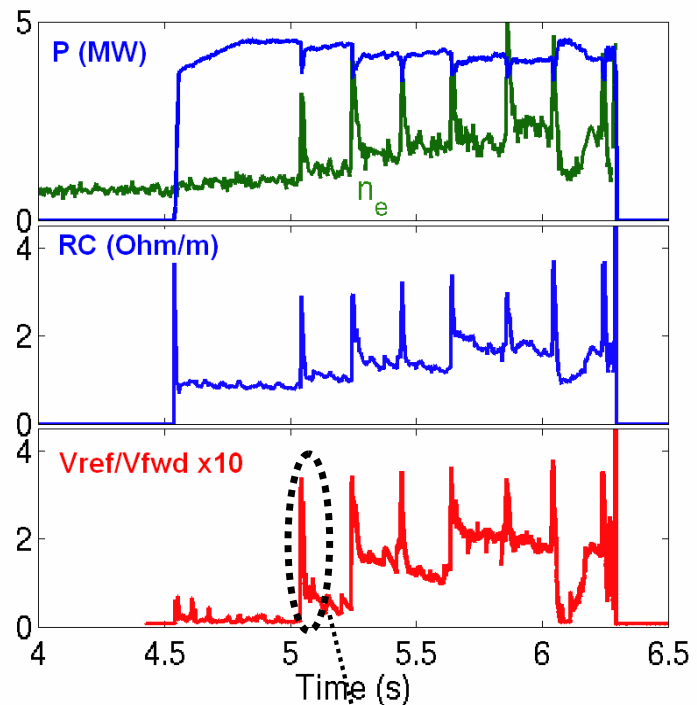

b)

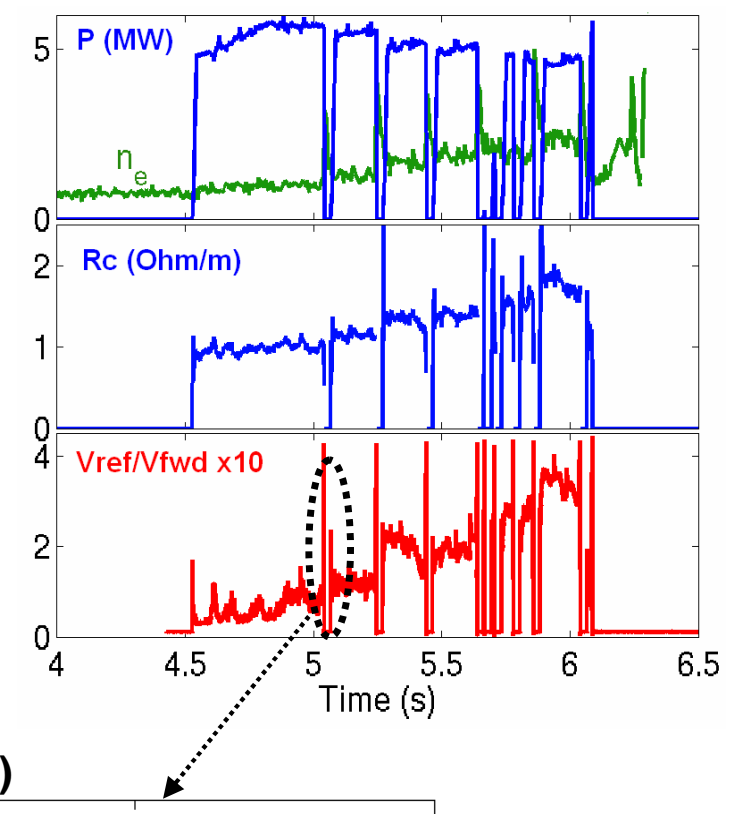

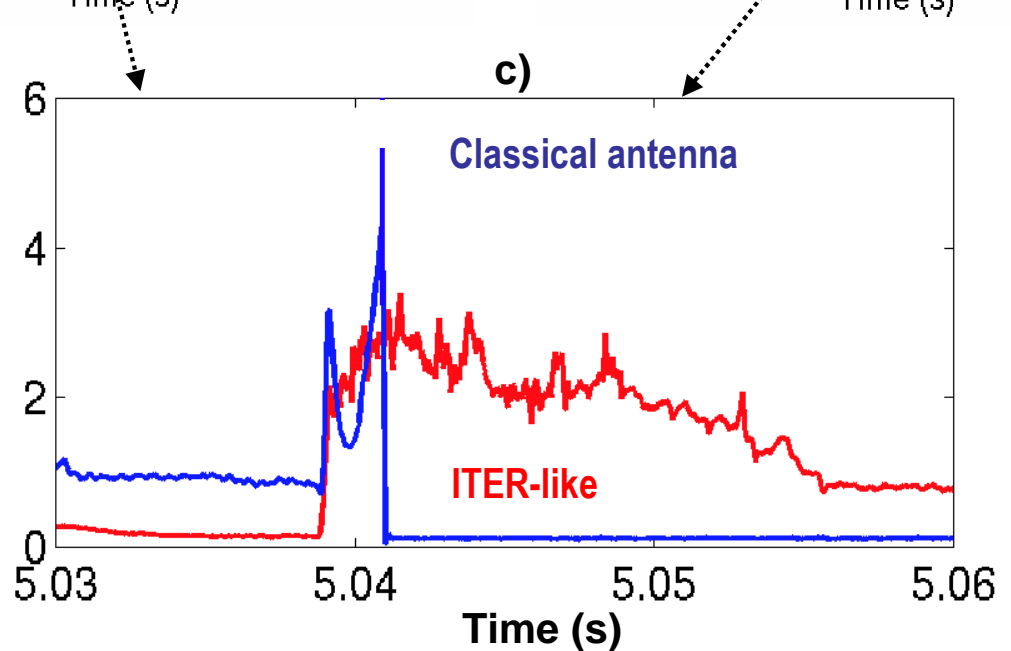

Figure 3: Behavior of the ILP antenna (a) during supersonic molecular beam injections, compared with the classical RDL antenna (b):

(a)\&(b) time traces of IC power (P in MW), plasma density at the last closed flux surface ( $\mathrm{n}_{\mathrm{e}}$ in $\left.1019 \mathrm{~m}-3\right)$, coupling resistance $\left(R_{c}\right.$ in $\left.\Omega / m\right)$, ratio $V_{\text {reflected }} / V_{\text {forward }}$ multiplied by 10 .

(c) zoom of the time evolution of $\mathrm{V}_{\text {reflected }} / \mathrm{V}_{\text {forward }}$ during a SMBI. 
Three days of the experimental campaign were used for the start-up phase under vacuum. In combination with vacuum conditioning, the vacuum settings of the matching elements (coaxial capacitors) of the launcher were slightly readjusted, compared to the known values in air at low power. During the start-up phase, the power and phase feedback controlled systems were also qualified and tested successfully. Starting with the capacitor values matched to vacuum conditions, and thanks to the electrical model predictions, the matching points for standard plasma (minority heating $\mathrm{D}(\mathrm{H}), 48 \mathrm{MHz}, \mathrm{B}_{\text {tor }}(\mathrm{R}=2.37 \mathrm{~m})=3.14 \mathrm{~T}$, line density $\mathrm{n}_{1}=4-5.10-19 \mathrm{~m}-2$ ) were reached after only few iterations on the 6 adjustable parameters of the antenna (the 4 capacitors settings and the phases of the 2 RF lines). When near such a "perfect" match point, the VSWR of the antenna was then optimized by balancing the voltages in each of the 4 straps and fine-tuning a toroidal $\pi$-phasing between the two half antennas.

With the ILP matching system set for chosen plasma conditions, the studies of the load resilience properties started with a series of shots at $48 \mathrm{MHz}$ and at a power level not exceeding $700 \mathrm{~kW}$. The first observation has been the low coupling resistance $\left(\mathrm{R}_{\mathrm{c}}\right)$ of the ILP compared to the RDL antenna. For similar plasma conditions and antenna position, where a $\mathrm{R}_{\mathrm{c}}$ of $4 \Omega . \mathrm{m}-1$ was measured on the classical antenna, the value on the ILP was around $1 \Omega . \mathrm{m}$ 1. The load tolerance studies were performed despite a rather small capability to vary the coupling resistance. Slow variations of $\mathrm{R}_{\mathrm{c}}$ from $0.3-1.8 \Omega . \mathrm{m}-1$ were firstly obtained by varying the plasma position (over a total range of $60 \mathrm{~mm}$ ) and the density in front of the antennas during RF pulses. With such rather steady conditions only a limited range of $R_{c}$ was accessible, due to the intrinsic difficulty of strongly modifying the edge density. To reach higher values of $R_{c}$ and push the investigation further, Supersonic Molecular Beam Injection (SMBI) was used. The Tore Supra SMB Injectors are able to launch a series of very short/dense gas jets at Mach number up to 5 [11]. The system injects gas at 3 different toroidal and poloidal locations. The use of SMBI allows the generation of fast perturbations (the transient time of the plasma edge density is inferior to 100 microseconds) in an L-mode environment and large excursions of the plasma density in front of the antenna; and so of the antenna coupling resistance. Figure 3 illustrates the comparative behavior between a standard antenna and the ILP during SMBI. During this given shot the density in front of the antenna (measured at $\mathrm{R}=3.1 \mathrm{~m}$ by a reflectometer) varies by a factor of 3 , and the corresponding transient coupling resistance $\mathrm{R}_{\mathrm{c}}$ ranges from 0.8 to $3 \Omega . \mathrm{m}-1$.

The agreement between the electrical models (RF circuit calculations that include 3D modeling for the complex structures) and the experimental measurements nevertheless appears to be in excellent agreement over the coupling resistance range. The Figure 4 illustrates this; the VSWR is plotted against the coupling resistance during a single plasma shot with plasma position varying (over a large range $60 \mathrm{~mm}$ ) and SMBI shot. Measurement covers respectively the low (plasma position variation) and the high parts (SMBI) of the $R_{c}$ value.

The ILP thus demonstrates a strong insensitivity to sudden changes of the edge density and coupling conditions, compared with the RDL antenna. From an operating point of view, the settings of the ILP matching array are also far less picky compared to the classical Tore Supra antenna. Indeed, at a given power level, while a variation of $\pm 0.5 \mathrm{pF}$ around the matching point are sufficient to mismatch the RDL, variations of $\pm 3 \mathrm{pF}$ on the ILP capacitors lead to strong voltage/current unbalance but do not prevent the coupling of the power to the plasma. 


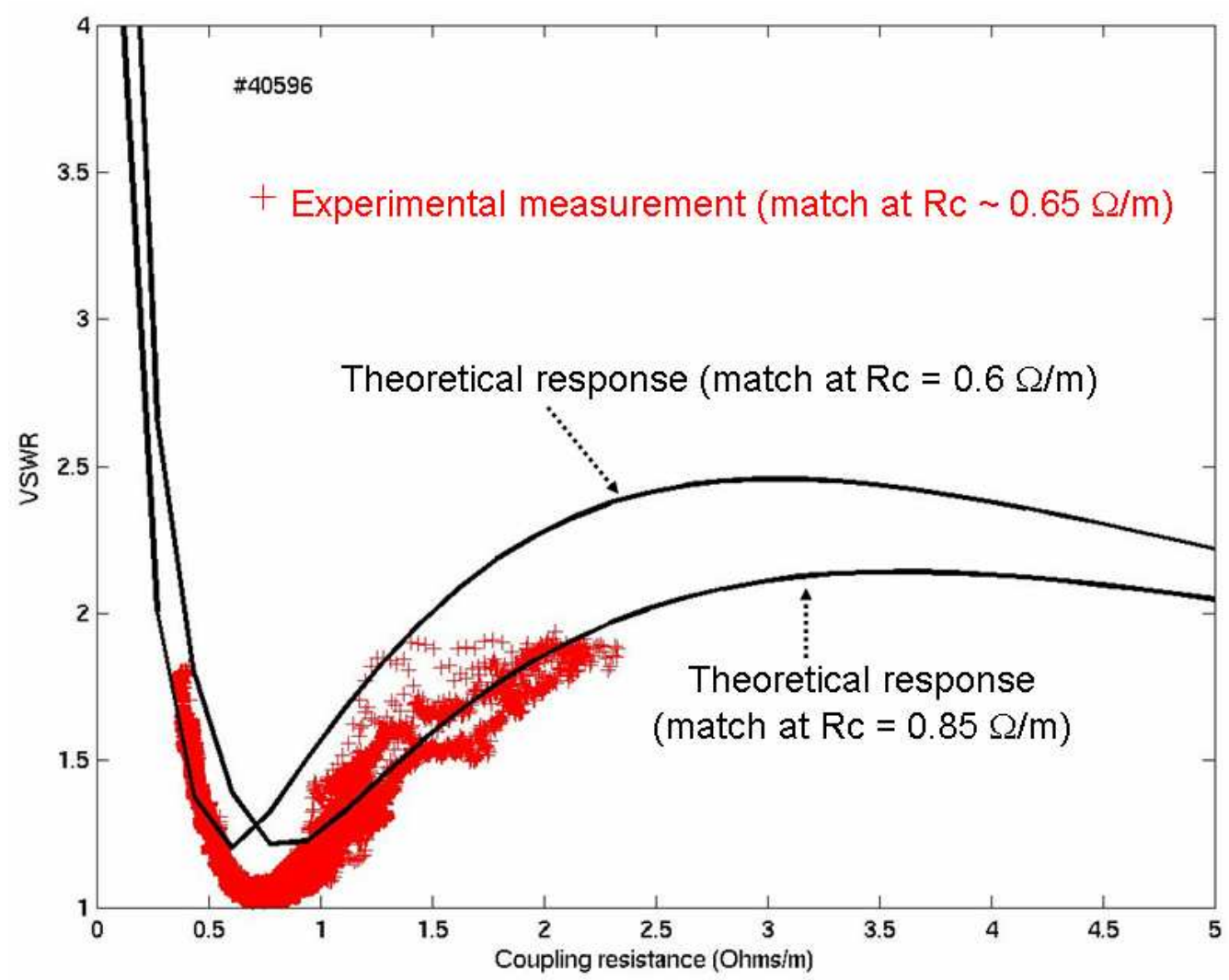

Figure 4: Shots 40596: VSWR as a function of the coupling resistance: experimental data from a single discharges (symbol: +); electrical simulation (bold lines).

\section{TECHNICAL LIMITATIONS}

The last part of the campaign was dedicated to technical tests of the antenna, with the objective to power the ILP up to its limits. The maximum power reached $2.2 \mathrm{MW}$ with $36 \mathrm{kV}$ on the straps. In order to reach such level of power, several hours of RF conditioning was necessary, process far longer on the ILP structure that features small conductors inter-spacing along the vacuum transmission line under vacuum, than on a RDL structure. Repetitive conditioning shots with power steps of $100 \mathrm{~kW}$ were required to reach the electrical limits. The only incident observed throughout all these repetition of pulses occurred at the very end of the campaign with a strong out gassing inside the impedance transformer: an incident that had triggered the closure of the vacuum valve.

\section{LESSONS TO BE LEARNED FOR ITER ARRAYS}

The ILP Tore Supra antenna is a so-called ITER relevant array, featuring 4 resonant symmetric straps, prototyping the more complex JET-EP antenna [12], using the same scheme but with 8 straps and at a second stage. The ITER IC launcher itself is powering 24 straps. However, despite the simplicity of its array, with only 6 adjustable parameters (generator phase \& power balance, 4 capacitor values), the ILP appears to be a suitable tool to validate the numerical models used as predictive tools to match the antennas. Even if the behavior of the ILP during ELMs cannot be assessed on Tore Supra, the robustness observed during fast SMBI variations of plasma conditions is encouraging news for future operation of IL arrays. The good performance of the phase and power control systems and the efficiency 
of the sub-harmonic arc detection also provide a key demonstration of the successful operation of such mandatory sub-systems.

\section{CONCLUSION}

The results obtained with the ITER-like prototype in Tore Supra represent a significant milestone on the way towards future integration of IC launchers on ITER. The operation of the ILP on 315 Tore Supra plasmas was successful from both a technical and physical points of view. Good behavior of the antenna subsystems, including the phase/power control and sub-harmonic arc detection systems, has been demonstrated. From a physical point view the robustness of an IL array under degraded and perturbed plasma coupling conditions has been validated.

The studies initiated with the ILP experiment will continue with the commissioning and operation of the JET-EP launcher, now installed on the JET device. This antenna will cover a larger range of experiments and features several specificities, common to the ITER array, in terms of dissymmetric Faraday screen with higher strap mutual-coupling, the presence of a second stage, complexity of the matching algorithm (real-time driven) and ,of course, operation with ELMs.

\section{REFERENCES}

[1] J.-M. Noterdaeme et al., g in Fusion Energy 1996 (Proc. 16th Int. Conf. Montreal, 1996), Vol. 3, p.335, IAEA, Vienna(1997).

[2] I. Monakhov et al, Fusion Engineering and Design 74, 467 (2005).

[3] K. Vulliez et al., Fusion Engineering and Design, Vol.66-68, 531 (2003).

[4] K. Vulliez et al., Fusion Engineering and Design, 74, 267 (2005).

[5] K. Vulliez et al., Proceedings of the $17^{\text {th }}$ RF Topical Conference on Radio Frequency Power in Plasmas, 119 (Clearwater, USA, 2007).

[6] G. Bosia et al., Proceedings of the $19^{\text {th }}$ IEEE/NPSS Symposium on Fusion Engineering, 138 (Atlantic City, USA, 2001).

[7] G.Bosia and S. Brémond, Proceedings of the $16^{\text {th }}$ Topical Conference on Radio Frequency Power in Plasmas, 174 (Park City, USA, 2005).

[8] K. Vulliez et al., Proceedings of the $23^{\text {rd }}$ Symposium on Fusion Technology, Venice, P3T -B-371 (2004).

[9] G. Berger-By et al., Fusion Engineering and Design 82 (2007) 716

[10] T. L. Owens et al., Proceedings of the $6^{\text {th }}$ Topical Conference on Radio Frequency Plasma Heating, 95 (1985).

[11] J. Bucalossi et al, in Fusion Energy 2002 (Proc. 19th Int. Conf., Lyon 2002) (2002)

(Vienna: IAEA) CD-ROM File EX/P4-04 and

http://www.iaea.org/programmes/ripc/physics/fec2002/html/fec2002.htm 